\title{
Recent Advances in the Photonic Integration of Mode-Locked Laser Diodes
}

\author{
K. Van Gasse $\left.{ }^{(}\right)$, S. Uvin, V. Moskalenko, S. Latkowski ${ }^{(}$, G. Roelkens $^{(}{ }^{\circledR}$, E. Bente ${ }^{\circledR}$, and B. Kuyken
}

\begin{abstract}
Mode-locked fiber and solid state lasers have played an essential role in several scientific and technological developments. The integration of mode-locked lasers on chips could enable their use in a wide range of applications. The advancement of semiconductor mode-locked laser diodes has been going on for several decades, but has recently seen the development of novel devices based on generic InP and III-V-on-silicon photonic integration platforms. These photonic integration platforms enable the use of standardized components and low-loss waveguides within the laser cavity, allowing for the design of advanced extended cavities. In this manuscript we give a review of these novel devices and compare their performance.
\end{abstract}

Index Terms-Semiconductor lasers, quantum well lasers, silicon photonics.

\section{INTRODUCTION}

$\mathbf{S}$ HORTLY after the first demonstrations of continuous wave lasers, the mode-locked operation of $\mathrm{He}-\mathrm{Ne}$ and Ruby lasers was achieved [1]. The domain has evolved rapidly since then. Notable milestones are the use of broadband gain media, such as Ti-Sapphire, that allow for generating sub 20 fs pulses [2] and later the development of fiber modelocked lasers (MLLs). The latter allowed for making more compact and practical devices. For a number of applications however, particularly in the field of microwave photonics and telecommunications even more compact devices are required. Here, one can think for example of semiconductor multiwavelength lasers where a large number of lines is imprinted with data that allow for Tbit/s datarates [3]. For these applications, the devices should be fabricated in high volumes at a relatively low cost and with a high yield. Chip-scale MLLs on silicon, or indium phosphide, could potentially fulfill these requirements. In this letter, we give an overview of the state-ofthe-art related to the development of on-chip MLLs, restricted to MLLs realized using Photonic Integrated Circuit (PIC) technology.

\section{Characteristics OF Mode-Locked LAsers}

In a MLL a non-linear mechanism is needed to phaselock the different lasing modes. In semiconductor lasers, mode-locking is generally achieved by using a saturable absorber (SA). The SA phase-locks the modes because it makes sure pulsed operation is favoured in the cavity, as high peak powers saturate the absorption and reduce the loss. When the pulse train produced by the MLL is sent to a photodiode several Radio Frequency (RF) signals, multiples of the repetition rate, are generated. The timing jitter of this pulse train results in phase noise of the RF signals, which can be substantial in some semiconductor MLLs. This large phase noise of the generated RF signals can limit the performance of the MLLs in applications such as microwave photonics. To improve the phase noise of the MLL, an external RF source can be used to modulate the SA and obtain hybrid mode-locking. However, in this review we will focus on MLLs operated without external reference, also referred to as passively MLLs.

Furthermore, the duration of the pulses, the width of the generated optical spectrum and the optical linewidth strongly depend on the type of mode-locking and laser architecture. Depending on the application, the laser design can be tailored to optimize a specific characteristic of the laser. For example in spectroscopy applications a wide output spectrum of the laser is required, consisting of a set of evenly spaced lines separated by the repetition frequency of the MLL. Other applications, such as coherent optical communication, require MLLs where the individual modes have a very narrow optical linewidth. In the following sections a number of MLL diodes and their key parameters such as the pulse duration, RF and optical line width, repetition frequency and optical bandwidth are discussed.

\section{MODE-LOCKED LASER DIODES}

A MLL diode can be realized using the same manufacturing techniques that are used for ridge waveguide laser diodes [7]. The main difference is the addition of a SA section, which can be achieved by electrically isolating a part of the gain waveguide and applying a reverse bias to it. An artistic rendering of such a semiconductor MLL is shown in Fig. 1(a). Most commonly, the laser cavity is created by using the cleaved facets of the ridge laser as mirrors. These lasers can produce short pulses of which the repetition rate is determined by the cavity length. Such lasers have been developed using many different III-V material platforms, ranging from GaAs/AlGaAs [7] to InP/InGaAsP or InP/AlGaInAs quantum wells (QWs) and InAs/InP quantum-dashes or quantumdots. Monolithic MLL diodes that generate sub-picosecond pulses have been reported on multiple material platforms, such as InP/AlGaInAs [4] and InAs/InP or InAs/GaAs quantum dots [8]. 


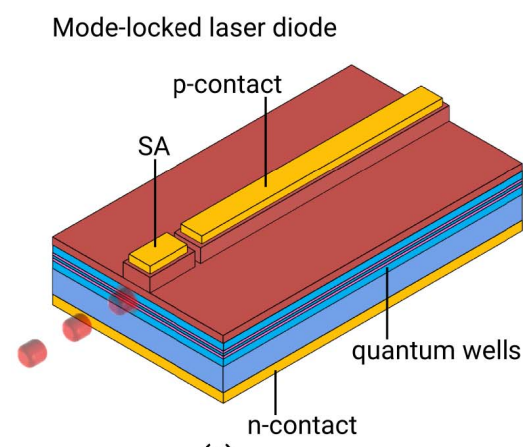

(a)

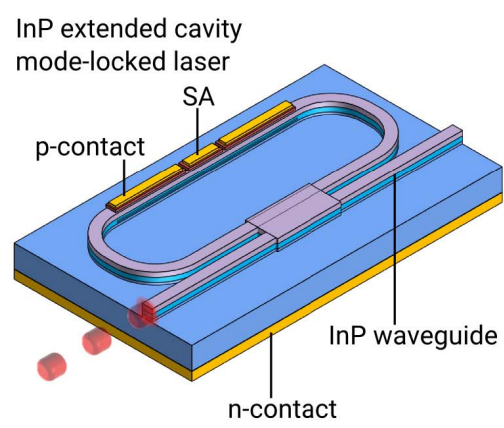

(b)

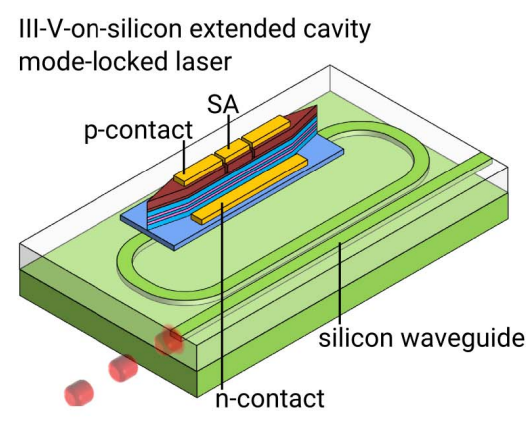

(c)

Fig. 1. Artistic rendering of different types of mode-locked laser diodes. (a) A monolithic quantum well laser diode as presented in [4]. (b) An extended cavity mode-locked laser diode realized using a generic InP photonic integration platform as reported in [5]. (c) An extended cavity ring-type mode-locked laser diode realized using a III-V-on-silicon photonic integration platform as presented in [6].

Both the RF linewidth of the repetition frequency and the optical linewidth reflect the noise of the laser. Very similar as in continuous wave lasers, the noise can be reduced by making use of extended cavity solutions. This can be achieved by adding a free space or fiber section to the laser cavity [9]. This idea is at the core of extended InP and III/V-on-silicon lasers and will be discussed in the following sections. With the advent of complex integrated photonic circuit platforms such as the active-passive InP platform [10] and the heterogeneous III-V-on-silicon platform [11], the external cavities can be realized on a single chip. Fig. 1(b) shows an InP extended cavity MLL, where passive InP waveguides take up the role of the passive external cavity. Fig. 1(c) shows a III-V-onsilicon MLL where the silicon waveguides take up this role. There are two other advantages of integrating MLLs using active-passive integration technologies. Firstly, the lasers can be combined with other circuits in which the generated pulses are used or manipulated. Secondly, it opens up a larger range of design possibilities for the lasers to improve performance. Such that one could approach the performance of free space and fibre-based extended cavity systems (e.g. [9]). The remainder of the letter will discuss these two approaches.

\section{INP EXTENDED CAVITY MODE-LOCKED LASERS}

The technology of photonic integration on InP has been used in industry for many years. It has developed to a level that complex monolithically integrated circuits can be realized. In this technology, the composition of the layer stack can be varied over the wafer using etching and regrowth techniques. This allows for the realization of photonic integrated circuits that are composed of ridge waveguide quantum-wellbased Semiconductor Optical Amplifiers (SOAs), passivewaveguide-based structures with different etch depths (e.g. multi-mode interferometers, arrayed waveguide gratings, splitters, distributed Bragg reflectors, etc.) and electro-optic phase modulators [10].

For example, it is appealing that fast electro-optical phase modulators can be integrated inside the laser cavity, allowing for precise control of the optical modes. This was demonstrated in a ring-type MLL with a repetition rate of $2.5 \mathrm{GHz}$ [12], where a long passive waveguide is used to achieve the relatively low repetition rate. Ring type lasers show very promising results and allow for making a laser cavity without on-chip reflectors, which can have an unwanted spectral response. Another example of a high-performance ring laser was presented in [5]. This laser produces pulses with a $900 \mathrm{fs}$ duration at a repetition rate of $20 \mathrm{GHz}$, resulting in a very wide coherent optical comb of $11.5 \mathrm{~nm}$. Ring-type lasers have shown to be able to generate even wider optical combs of up to $40 \mathrm{~nm}$, as reported in [13]. More advanced ring lasers topologies were demonstrated using InP photonic integration technology. For example, a ring laser with two SAs inside the cavity offered control of the lasing direction and reduced feedback sensitivity [14].

Compact broadband on-chip reflectors have been developed in the form of Multimode Interference Reflectors (MIRs). Using these on-chip broadband reflectors several lasers with advanced linear cavities and hybrid cavities have been realized. Using a colliding pulse MIR linear cavity, the lowest repetition rate InP passively MLL was realized with a repetition rate of $1 \mathrm{GHz}$ [15]. A more complex linear cavity laser with a fundamental repetition rate of $25 \mathrm{GHz}$ was demonstrated also using MIRs [16]. This laser cavity contained 3 SAs, that allowed to obtain harmonic mode-locking. Furthermore, the laser produced short (500 fs) pulses with a high $(100 \mathrm{GHz})$ repetition rate. The use of cavities with multiple SAs also enables the systematic study of the SA positions influence on the MLL performance. A laser with 2 SAs inside the cavity was used to determine the difference in performance caused by placing the SA either by the low or high-reflection mirror of a MLL [17]. It was found that placing the SA at the low-reflection mirror (anti-colliding pulse) improves the performance compared to when the SA is placed at the high-reflection mirror. The InP photonic integration platform also offers components such as Distributed Bragg Reflectors (DBRs) of which the central wavelength is tunable. DBRs can be realized by periodically varying the refractive index of a passive waveguide. Such tunable reflectors can be used as a mirror for the MLL cavity, which allows to control the wavelength at which the laser operates. Due to the spectral dependence of the SOA gain and SA absorption, the performance of the MLL depends on the lasing wavelength. In [18] it 
was shown that by tuning the DBR reflection towards shorter wavelengths, shorter pulse duration and lower RF linewidth can be achieved.

Other laser cavities with increased complexity were explored, such as a ring cavity where reflectors are placed inside the cavity to realize harmonic mode-locking [19]. This laser presented mode-locking operation with repetition rates ranging from $50 \mathrm{GHz}$ to $450 \mathrm{GHz}$ by varying the bias conditions.

As explained above, the use of photonic integration platforms allows one to combine the laser with other on-chip components. For example, a MLL with a pulse-picker and power amplifier on the same PIC were demonstrated [20].

\section{III-V-ON-SILICON MODE-LOCKED LASERS}

Heterogeneous III-V-on-silicon photonic integrated circuits have progressed enormously and are becoming a mature technology platform [11]. In this approach a III-V gain material, epitaxially grown on their native substrate, is transferred on top of a silicon passive photonic integrated circuit through bonding [11] or micro-transfer printing [21].

The first demonstration of III-V-on-silicon MLLs was reported in [22] where two linear cavity devices with an AlGaInAs amplifier were presented. This was quickly followed by a ring-type III-V-on-silicon MLL with a $30 \mathrm{GHz}$ repetition rate [23]. These first demonstrations did not have beyond state-of-the-art performance, but illustrated the feasibility of the technology.

These first results were quickly followed by several III-V-on-silicon MLLs based on a InGaAsP QW gain section and low-loss silicon waveguides $(0.7 \mathrm{~dB} / \mathrm{cm})$ [6]. These devices showed improved performance, as they combined a narrow RF linewidth with a wide optical comb. For example, a colliding-pulse Fabry-Perot MLL produced 2 ps pulses at a repetition rate of $4.7 \mathrm{GHz}$ with a pulse energy of $600 \mathrm{fJ}$ [24]. Furthermore, the laser had a RF linewidth of $12 \mathrm{kHz}$ and a wide optical spectrum of almost $9 \mathrm{~nm}$. As mentioned in the previous section, anti-colliding pulse cavities provide excellent performance and this was also demonstrated using the III-V-on-silicon technology [6]. This device with a repetition rate of $5 \mathrm{GHz}$ and an RF linewidth of $2 \mathrm{kHz}$, generates $1 \mathrm{pJ}$ pulses with a 3 ps pulse duration.

Furthermore, the low-loss silicon optical waveguides allow to realize on-chip lasers with a low repetition rate and narrow optical linewidth. For example in [25] a ring-type passively MLL with a repetition rate of $2 \mathrm{GHz}$ was demonstrated. The laser produced a $15 \mathrm{kHz} \mathrm{RF}$ linewidth and an optical comb spanning $1 \mathrm{~nm}$, significantly outperforming the $1 \mathrm{GHz}$ InP PIC laser [15]. Furthermore, different types of high-performance filters can be added to the cavity using passive silicon photonic components. For example, a $20 \mathrm{GHz}$ MLL was realized by adding a ring filter to the cavity of a $2 \mathrm{GHz}$ MLL. This laser showed a ten times narrower optical linewidth $(1 \mathrm{MHz})$ than a similar MLL with a $20 \mathrm{GHz}$ cavity without ring filter [26]. This is an important advantage of this technology as other semiconductor MLL diodes often have linewidth of several $\mathrm{MHz}$, making them unsuited for several applications such as coherent communication.
TABLE I

Overview of MLL Diodes ReAlized on DifFERENT PlatForms

\begin{tabular}{|l|l|l|l|l|l|l|l|}
\hline Ref. & $\begin{array}{l}f_{r e p} \\
(\mathrm{GHz})\end{array}$ & $\begin{array}{l}\tau_{p} \\
(\mathrm{ps})\end{array}$ & $\begin{array}{l}E_{p} \\
(\mathrm{pJ})\end{array}$ & $\begin{array}{l}\Delta f_{R F} \\
(\mathrm{kHz})\end{array}$ & $\begin{array}{l}\Delta \lambda \\
(\mathrm{nm})\end{array}$ & $\begin{array}{l}\Delta f_{\text {opt }} \\
(\mathrm{MHz})\end{array}$ & nom \\
\hline$[8]-(1)$ & 92 & 0.3 & 0.1 & - & 11.7 & - & 17 \\
\hline$[4]-(1)$ & 40 & 0.7 & $<0.1$ & 100 & 5.5 & - & 17 \\
\hline$[3]-(1)$ & 20 & 5 & 0.5 & 1.8 & 6.1 & 10 & 58 \\
\hline$[5]-(2)$ & 20 & 0.9 & $<0.1$ & 2400 & 11.5 & 900 & 72 \\
\hline$[19]-(2)$ & 100 & 0.5 & $<0.1$ & - & 5.7 & - & 7 \\
\hline$[27]-(3)$ & 1 & 7 & 0.5 & 0.4 & 10 & 0.3 & 1000 \\
\hline$[31]-(3)$ & 20 & 0.9 & 0.1 & 1.1 & 3 & - & 18 \\
\hline
\end{tabular}

The lowest repetition rate for an on-chip passively MLL is $1 \mathrm{GHz}$. This was realized in III-V-on-silicon using an anticolliding pulse design [27]. This device produces 7 ps pulses with an estimated pulse energy of $500 \mathrm{fJ}$. Furthermore, an RF linewidth of $450 \mathrm{~Hz}$ and a optical spectrum wider than $10 \mathrm{~nm}$ (10 dB bandwidth) were reported. This is interesting, because it is the first time an on-chip laser produces more than an 1000 optical modes. Furthermore, the optical linewidth of the individual optical modes is estimated to be around $250 \mathrm{kHz}$, at the center lasing wavelength.

External feedback is another approach to improve the RF or optical linewidth of a MLL diode. In [28] a $17 \mathrm{GHz}$ colliding-pulse Fabry-Perot III-V-on-silicon MLL was presented with an optical wavelength of $1.3 \mu \mathrm{m}$. A long $(4 \mathrm{~cm})$ silicon waveguide delay line on the same chip allowed to feedback a part of the laser output to the cavity. Using this type of feedback the RF linewidth of the device was reduced from $44 \mathrm{kHz}$ to $29 \mathrm{kHz}$. In the same letter the influence of the number of QWs was investigated by fabricating the device with both 3 and 5 QWs. It was found that using 3 QWs greatly reduces the RF linewidth due to the reduced amount of ASE coupling to the laser cavity.

Although most of the previously described devices show promising performance, the pulse energy is too low for several applications. Therefore, several high-power III-V-onsilicon amplifiers were designed and fabricated. Both a $3 \mathrm{QW}$ AlGaInAs [29] and 6 QW InGaAsP amplifier [30] were developed with a saturated output power in the $50 \mathrm{~mW}$ range. Using the high power $3 \mathrm{QW}$ amplifier, a III-V-on-silicon Fabry-Perot $20 \mathrm{GHz}$ MLL was fabricated. This MLL generated pulses with a pulse peak power of $100 \mathrm{~mW}$. Furthermore, the produced pulses had an estimated pulse duration of $0.9 \mathrm{ps}$, which is the shortest pulse measured from a III-V-on-silicon MLL [31]. The device also had a record narrow RF linewidth $(1.1 \mathrm{kHz})$ for a $20 \mathrm{GHz}$ III-V-on-silicon MLL. However, the optimal performance of the MLL happens at $2 \mathrm{~mW}$ of average output power $(0.1 \mathrm{pJ}$ pulse energy). For many applications a pulse energy exceeding $5 \mathrm{pJ}$ would be very interesting, as this would allow on-chip spectral broadening using non-linear waveguides. To achieve this goal further progress is needed in both the cavity design and amplifier performance.

One possibility to increase the pulse energy would be to use quantum dots, as they have lower confinement factors and a wide gain bandwidth. A III-V-QD-on-silicon MLL with promising results was recently demonstrated [32].

Another appealing option is to use silicon nitride waveguides, as they allow for dispersion engineering and have no two-photon absorption. No III-V-on-silicon-nitride 
was demonstrated so far, but recently a MLL based on a buttcoupled silicon nitride cavity was reported [33].

\section{CONCLUSION}

In table I, an overview of lasers from different platforms is given. The left column of the table indicates the reference and the platform used to realize the laser, (1) monolithic III-V, (2) InP photonic integration platform and (3) III-V-on-silicon. The other columns show the relevant parameters of the devices: repetition rate $\left(f_{\text {rep }}\right)$, pulse duration $\left(\tau_{p}\right)$, pulse energy $\left(E_{p}\right)$, RF linewidth $\left(\Delta f_{R F}\right)$, optical bandwidth $(\Delta \lambda)$, optical linewidth $\left(\Delta f_{o p t}\right)$ and the total number of optical modes in the comb (nom).

From the data presented in table I we can conclude that all discussed platforms can deliver high-performance integrated MLLs in terms of pulse duration and optical bandwidth. However, the InP photonic integration platform offers extensive design options when compared to the other platforms. This, because both active and passive components can be easily integrated inside the cavity. When compared to the InP platform, the III-V-on-silicon approach offers lower loss silicon waveguides. These can be used to achieve low $(1 \mathrm{GHz})$ repetition rate MLLs and MLLs with a narrow optical linewidth. Although each platform has its own advantages, they all suffer from a limited pulse power $(1 \mathrm{pJ})$. To further improve the performance of integrated MLLs, new amplifier and waveguide designs will be needed with better power handling. This would enable the use of integrated MLL in a wide range of applications such as sensing, optical communications and microwave photonics.

\section{REFERENCES}

[1] K. Gurs, "Beats and modulation in optical ruby-masers," in Quantum Electronics. New York, NY, USA: Columbia Univ. Press. 1964.

[2] M. T. Asaki, C.-P. Huang, D. Garvey, J. Zhou, H. C. Kapteyn, and M. M. Murnane, "Generation of 11-fs pulses from a self-mode-locked Ti: Sapphire laser," Opt. Lett., vol. 18, no. 12, pp. 977-979, 1993.

[3] S. Liu et al., "High-channel-count $20 \mathrm{GHz}$ passively mode-locked quantum dot laser directly grown on $\mathrm{Si}$ with $4.1 \mathrm{Tbit} / \mathrm{s}$ transmission capacity," Optica, vol. 6, no. 2, pp. 128-134, 2018.

[4] J. H. Marsh and L. Hou, "Mode-locked laser diodes and their monolithic integration," IEEE J. Sel. Topics Quantum Electron, vol. 23, no. 6, Nov./Dec. 2017, Art. no. 1100611.

[5] V. Moskalenko, S. Latkowski, S. Tahvili, T. de Vries, M. Smit, and E. Bente, "Record bandwidth and sub-picosecond pulses from a monolithically integrated mode-locked quantum well ring laser," Opt. Express, vol. 22 , no. 23 , pp. 28865-28874, Nov. 2014.

[6] S. Keyvaninia et al., "III-V-on-silicon anti-colliding pulse-type modelocked laser," Opt. Lett., vol. 40, no. 13, pp. 3057-3060, Jul. 2015.

[7] E. A. Avrutin, J. H. Marsh, and E. L. Portnoi, "Monolithic and multigigahertz mode-locked semiconductor lasers: Constructions, experiments, models and applications," IEE Proc.-Optoelectron., vol. 147, no. 4, pp. 251-278, Aug. 2000

[8] Z. Lu, J. R. Liu, S. Raymond, P. J. Poole, P. J. Barrios, and D. Poitras, "312-fs pulse generation from a passive C-band InAs/InP quantum do mode-locked laser," Opt. Express, vol. 16, no. 14, pp. 10835-10840, 2008.

[9] P. J. Delfyett, A. Klee, K. Bagnell, P. Juodawlkis, J. Plant, and A. Zaman, "Exploring the limits of semiconductor-laser-based optical frequency combs," Appl. Opt., vol. 58, no. 13, pp. D39-D49, May 2019.

[10] M. Smit et al., "An introduction to InP-based generic integration technology," Semicond. Sci. Technol., vol. 29, no. 8, 2014, Art. no. 083001.

[11] T. Komljenovic, D. Huang, P. Pintus, M. A. Tran, M. L. Davenport, and J. E. Bowers, "Photonic integrated circuits using heterogeneous integration on silicon," Proc. IEEE, vol. 106, no. 12, pp. 2246-2257, Dec. 2018.
[12] S. Latkowski et al., "Monolithically integrated $2.5 \mathrm{GHz}$ extended cavity mode-locked ring laser with intracavity phase modulators," Opt. Lett., vol. 40, no. 1, pp. 77-80, Jan. 2015.

[13] V. Moskalenko, J. Koelemeij, K. Williams, and E. Bente, "Study of extra wide coherent optical combs generated by a QW-based integrated passively mode-locked ring laser," Opt. Lett., vol. 42, no. 7, pp. 1428-1431, 2017.

[14] L. Nielsen, E. A. J. M. Bente, E. den Haan, and M. J. R. Heck, "Theoretical and experimental investigation of unidirectionality in an integrated semiconductor ring mode-locked laser with two saturable absorbers," IEEE J. Quantum Electron, vol. 54, no. 5, Oct. 2018, Art. no. 2000810.

[15] R. Guzmán, C. Gordon, L. Orbe, and G. Carpintero, "1 GHz InP onchip monolithic extended cavity colliding-pulse mode-locked laser," Opt. Lett., vol. 42, no. 12, p. 2318, Jun. 2017.

[16] M.-C. Lo, R. Guzmán, and G. Carpintero, "Femtosecond pulse and terahertz two-tone generation from facet-free multi-segment laser diode in InP-based generic foundry platform," Opt. Express, vol. 26, no. 14, pp. 18386-18398, Jul. 2018.

[17] V. Moskalenko, K. A. Williams, and E. A. J. M. Bente, "Integrated Extended-Cavity $1.5-\mu \mathrm{m}$ semiconductor laser switchable between selfand anti-colliding pulse passive mode-locking configuration," IEEE J. Sel. Topics Quantum Electron, vol. 21, no. 6, Nov./Dec. 2015, Art. no. 1101306.

[18] V. Moskalenko, K. A. Williams, and E. A. J. M. Bente, "Pulse narrowing and RF linewidth reduction of integrated passively mode-locked laser in anticolliding design by means of spectral tuning," IEEE Photon. J., vol. 8, no. 4, Aug. 2016, Art. no. 1502810.

[19] M.-C. Lo, R. Guzmán, and G. Carpintero, "InP femtosecond modelocked laser in a compound feedback cavity with a switchable repetition rate," Opt. Lett., vol. 43, no. 3, pp. 507-510, Feb. 2018.

[20] X. Guo, A. H. Quarterman, A. Wonfor, R. V. Penty, and I. H. White, "Monolithically integrated tunable mode-locked laser diode source with individual pulse selection and post-amplification," Opt. Lett., vol. 41, no. 20, pp. 4835-4838, Oct. 2016.

[21] J. Zhang et al., "III-V-on-si photonic integrated circuits realized using micro-transfer-printing," APL Photon., to be published.

[22] B. R. Koch, A. W. Fang, O. Cohen, and J. E. Bowers, "Mode-locked silicon evanescent lasers," Opt. Express, vol. 15, no. 18, pp. 11225-11233, 2007.

[23] A. W. Fang et al., "A racetrack mode-locked silicon evanescent laser," Opt. Express, vol. 16, no. 2, pp. 1393-1398, 2008.

[24] S. Keyvaninia et al., "Narrow-linewidth short-pulse III-V-on-silicon mode-locked lasers based on a linear and ring cavity geometry," Opt. Express, vol. 23, no. 3, pp. 3221-3229, 2015.

[25] S. Srinivasan et al., "Harmonically mode-locked hybrid silicon laser with intra-cavity filter to suppress supermode noise," IEEE J. Sel. Topics Quantum Electron, vol. 20, no. 4, Jul./Aug. 2014, Art. no. 1100208.

[26] S. Srinivasan et al., "Low phase noise hybrid silicon modelocked lasers," Frontiers Optoelectron., vol. 7, no. 3, pp. 265-276, 2014.

[27] Z. Wang et al., "A III-V-on-Si ultra-dense comb laser," Light, Sci. Appl., vol. 6, no. 5, May 2017, Art. no. e16260.

[28] S. Srinivasan, E. Norberg, T. Komljenovic, M. Davenport, G. Fish, and J. E. Bowers, "Hybrid silicon colliding-pulse mode-locked lasers with on-chip stabilization," IEEE J. Sel. Topics Quantum Electron, vol. 21, no. 6, Nov./Dec. 2015, Art. no. 1101106.

[29] M. L. Davenport, S. Skendžić, N. Volet, J. C. Hulme, M. J. R. Heck, and J. E. Bowers, "Heterogeneous silicon/III-V semiconductor optical amplifiers," IEEE J. Sel. Topics Quantum Electron, vol. 22, no. 6, Nov./Dec. 2016, Art. no. 3100111.

[30] K. Van Gasse, R. Wang, and G. Roelkens, "27 dB gain III-V-on-silicon semiconductor optical amplifier with $>17 \mathrm{dBm}$ output power," Opt. Express, vol. 27, no. 1, pp. 293-302, Jan. 2019.

[31] M. L. Davenport, S. Liu, and J. E. Bowers, "Integrated heterogeneous silicon/III-V mode-locked lasers," Photon. Res., vol. 6, no. 5, pp. 468-478, May 2018.

[32] G. Kurczveil, C. Zhang, A. Descos, D. Liang, M. Fiorentino, and R. Beausoleil, "On-chip hybrid silicon quantum dot comb laser with 14 error-free channels," in Proc. IEEE Int. Semiconductor Laser Conf. (ISLC), Sep. 2018, pp. 1-2.

[33] J. Mak et al., "Linewidth narrowing via low-loss dielectric waveguide feedback circuits in hybrid integrated frequency comb lasers," Opt. Express, vol. 27, no. 9, pp. 13307-13318, 2019. 